\title{
Evaluation of Sexual Dimorphism in Hypothalamus of Chicken (Gallus domesticus)
}

\author{
Arkaprabha Shee ${ }^{1 *}$, Partha Das ${ }^{1}$, Sagun Saren $^{1}$, Palash Biswas ${ }^{1}$, \\ Aniruddha Banerjee ${ }^{2}$ and Md. Mofijul Islam ${ }^{1}$ \\ ${ }^{1}$ Department of Veterinary Anatomy and Histology, ${ }^{2}$ Department of Veterinary Pathology, \\ Faculty of Veterinary and Animal Sciences, WBUAFS, Kolkata-700037, India \\ *Corresponding author
}

\section{A B S T R A C T}

Keywords

Chicken,

Hypothalamus,

Preoptic nucleus,

Suprachiasmatic

nucleus, Sexual

Dimorphism

Article Info

Accepted:

04 June 2018

Available Online:

10 July 2018
The present study was conducted to identify sexually dimorphic regions in the chicken hypothalamus. Brain samples of apparently healthy chicken of either sex of 6-7 weeks was processed and consecutively stained by Hematoxylin and Eosin, Cresyl Violet and Toluidine Blue. The length and breadth of selected nuclei (preoptic nucleus, periventricular preoptic nucleus, medial suprachiasmatic nucleus and lateral hypothalamic nucleus) was calculated by Leica Qwin Images Analyser software in Leica DM 2000 Microscope. The study suggested that preoptic and medial suprachiasmatic nuclei were significantly $(P<0.01)$ larger in males than females and also demonstrated that in Nissl stained sections the Preoptic nucleus appeared longer and stained more intensely in males than in females.

\section{Introduction}

Hypothalamus is the ventral portion of diencephalon on either side of third ventricle and it has been implicated in a variety of physiological mechanisms and behavioural responses including adenohypophyseal regulation (Halasz and Pupp, 1965), especially Gonadotrophin release (Gorski, 1968; Wiegand and Terasawa, 1982), thermoregulation (Squires and Jacobsen, 1968; Day et al., 1979) and hypovolemic thrist (Swanson et al., 1978).
Avian hypothalamus (Fig. 1) has a significant role in the control of avian sexual behaviour. Several morphological sex differences have been identified in and around the hypothalamus of rodent brain (Raisman and Field, 1973; Greenough et al., 1977; Gorski et al., 1978).

Due to paucity of literature the present study has been undertaken to identify sexually dimorphic regions in the chicken hypothalamus. 


\section{Materials and Methods}

\section{Place of study}

The present study was carried out at the Department of Veterinary Anatomy and Histology, West Bengal University of Animal and Fishery Sciences, Kolkata.

\section{Source of the samples}

20 male and 20 female skulls of apparently healthy chicken of 6-7 weeks was collected from local slaughter house within Kolkata for the period from June 2016 to May 2017.

\section{Histological studies}

Brain samples were carefully dissected out and preserved in 10\% NBF for further studies. Selected area of brains was processed and consecutively stained by Hematoxylin and Eosin, Cresyl Violet and Toluidine Blue staining method as per the procedure prescribed by Suvarna et al., (2012).

The sections were taken at 10-15 $\mu \mathrm{m}$ thickness with the help of Leica rotary microtome.

\section{Morphometrical analysis}

The length and breadth of selected nuclei of hypothalamus was calculated by Leica Qwin Images Analyser software in Leica DM 2000 Microscope.

\section{Statistical analysis}

All the data were statistically analysed by unpaired t-test. The significance ( $P$ value) was recorded at $1 \%$ level $(P<0.01)$. Statistical analysis of collected data was performed using statistical software (GraphPad Prism 3.00; GraphPad Software, San Diego, California, USA).

\section{Results and Discussion}

The Chicken hypothalamus formed the ventral portion of diencephalon on either side of third ventricle (Fig. 1). It had been divided into a large number of more or less distinct cell groups and it was traversed by several major fibre systems. In the present study a large oval shaped cellular aggregation was found at dorsal angel of lateral ventricle in coronal section in the preoptic region. The morphological appearance of this cellular aggregation represented the pre optic nucleus (PON) (Fig. 2). A separate cellular aggregation was situated between the PON and medial suprachiasmatic nucleus ( $\mathrm{mSCN})$, on either side of the lateral border of lateral ventricle. This nucleus is termed as periventricular preoptic nucleus (PPN) (Fig. $3)$. A paired cell groups was clearly observed at the height of the ventral portion of the periventricular preoptic nucleus at the level of lateral angle of third ventricle interconnected by a small sub-ventricular queue of neurons. This aggregation is termed as medial suprachiasmatic nucleus (mSCN) (Fig. 4). An aggregation of neuron was found lateral to the $\mathrm{mSCN}$ at the dorsal border of the optic chiasma. In the further rostro-caudal section these aggregations revealed extension of the increase of cell group in size and extended into the lateral hypothalamic area. This cellular aggregation represented the lateral hypothalamic nucleus (LHN) (Fig. 5).

The present study demonstrated that PON (Fig. 6 and 7) was significantly $(P<0.01)$ larger in males than females (Table 1). The PON was continuous with the periventricular preoptic nucleus (PPN). Further the PPN was extended ventrally to the mSCN. In birds Panzica et al., (1987, 1996) identified the similar observation. They showed that the medial preoptic nucleus (POM) that was significantly larger in adult male than in adult female quail. 
The volumetric difference of the POM resulted from a difference in the adult hormonal milieu of males and females (activational effect). In contrast, the size of neurons in the dorsolateral part of POM appeared to be irreversibly affected by embryonic steroids and this feature was therefore a better to correlate the behavioural sex difference. The POM was bidirectionally connected to many brain areas. It received inputs from a variety of sensory areas and formed a number of regulatory areas (e.g., catecholaminergic cell groups). This is in accordance with our present findings. Other research findings reported that one of the preoptic nuclei was larger in male Japanese quail than in females based on an earlier study of the pigeon brain, this area was named nucleus preopticusmedialis (POM) (Watson and Regan, 1989) which also in agreement with our result.

Results of the current study showed that length and breadth of the mSCN nucleus was significantly $(P<0.01)$ greater for male than for female (Table 1). Similar observation was made by Robinson et al., (1986). They performed computer-assisted morphometric techniques to demonstrate sexual dimorphism in SD rat and documented that mean volume of the SCN was $26 \%$ greater in males than females.

Table.1 Morphometrical analysis of hypothalamic nucleus in chicken

\begin{tabular}{|l|l|l|l|}
\hline $\begin{array}{l}\text { Hypothalamic } \\
\text { Nucleus }\end{array}$ & $\begin{array}{c}\text { Male } \\
(\text { Mean } \pm \text { SE) }\end{array}$ & $\begin{array}{c}\text { Female } \\
(\text { Mean } \pm \text { SE) }\end{array}$ & $P$ Value \\
\hline $\begin{array}{l}\text { PON } \\
\text { Length }(\boldsymbol{\mu m}) \\
\text { Breadth }(\boldsymbol{\mu m})\end{array}$ & $\begin{array}{l}570.55 \pm 0.23 \\
238.48 \pm 0.018\end{array}$ & $\begin{array}{l}540.38 \pm 6.50 \\
217.42 \pm 4.02\end{array}$ & $P<0.01$ \\
\hline $\begin{array}{l}\text { PPN } \\
\text { Length }(\boldsymbol{\mu m})\end{array}$ & $371.6 \pm 2.92$ & $368.00 \pm 0.08$ & $P>0.01$ \\
Breadth $(\boldsymbol{\mu m})$ & $337.4 \pm 123$ & $334.30 \pm 1.34$ & \\
\hline $\begin{array}{l}\text { mSCN } \\
\text { Length }(\boldsymbol{\mu m})\end{array}$ & $458.3 \pm 2.78$ & $449.53 \pm 0.05$ & $P<0.01$ \\
Breadth $(\boldsymbol{\mu m})$ & $350.2 \pm 0.06$ & $336.3 \pm 4.83$ & \\
\hline $\begin{array}{l}\text { LHN } \\
\text { Length }(\boldsymbol{\mu m})\end{array}$ & $952.21 \pm 4.02$ & $946.42 \pm 4.21$ & $P>0.01$ \\
Breadth $(\boldsymbol{\mu m})$ & $655.41 \pm 1.64$ & $652.29 \pm 2.32$ & \\
\hline
\end{tabular}



Figure.1 Photomicrograph showing hypothalamus of chicken; Toluidine Blue X 4. (C) PON, (B) PPN, (A) mSCN (D) Lateral ventricle (E) Third ventricle 


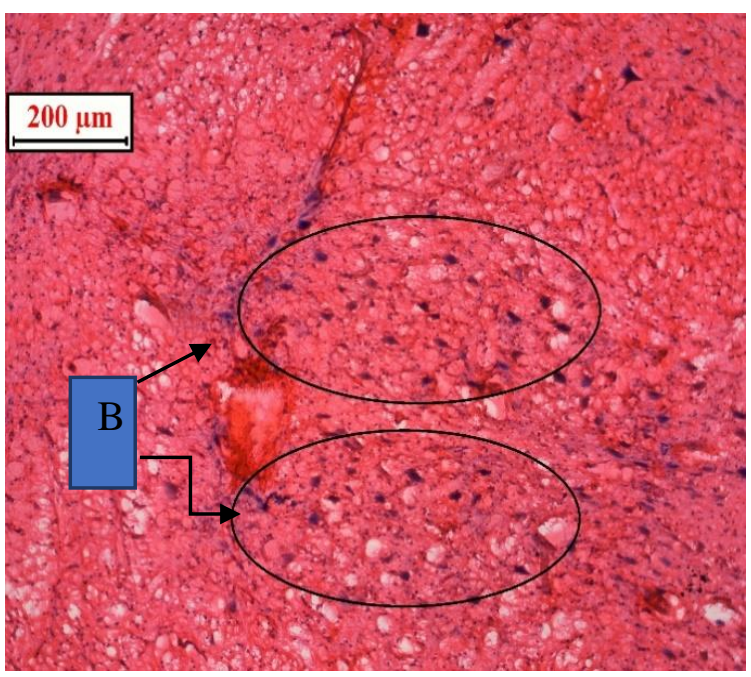

Figure.2 Photomicrograph showing PON, H\&E X 10. (B) PON

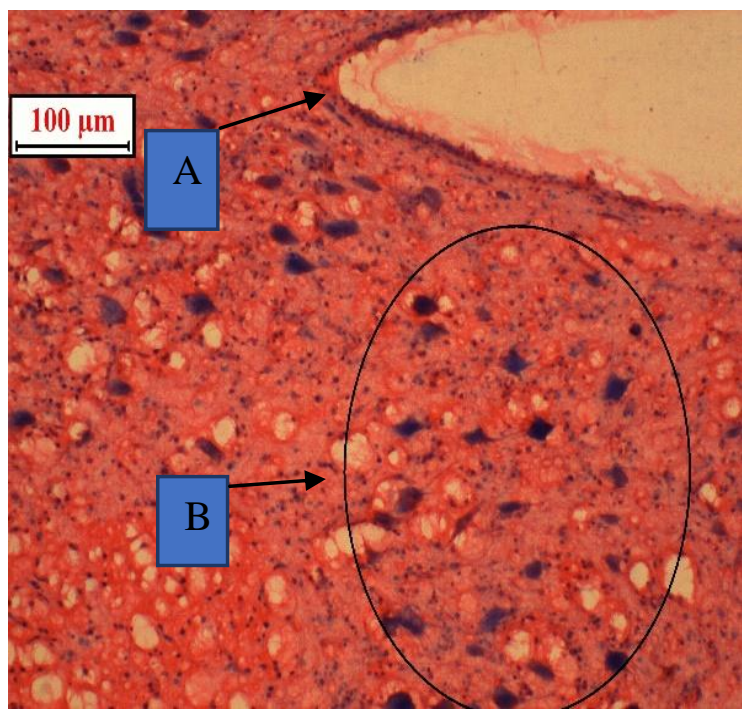

Figure.3 Photomicrograph showing PPN H\&E X 20; (A) Dorsal angel of lateral ventricle (B) PPN

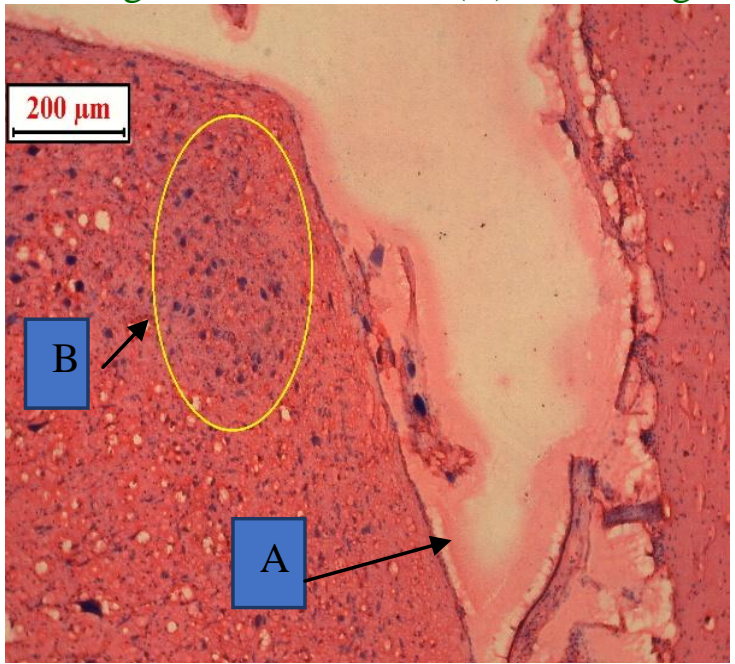

Figure.4 Photomicrograph showing mSCN H\&E X 10 . (A) Lateral angle of third ventricle (B) $\mathrm{mSCN}$ 


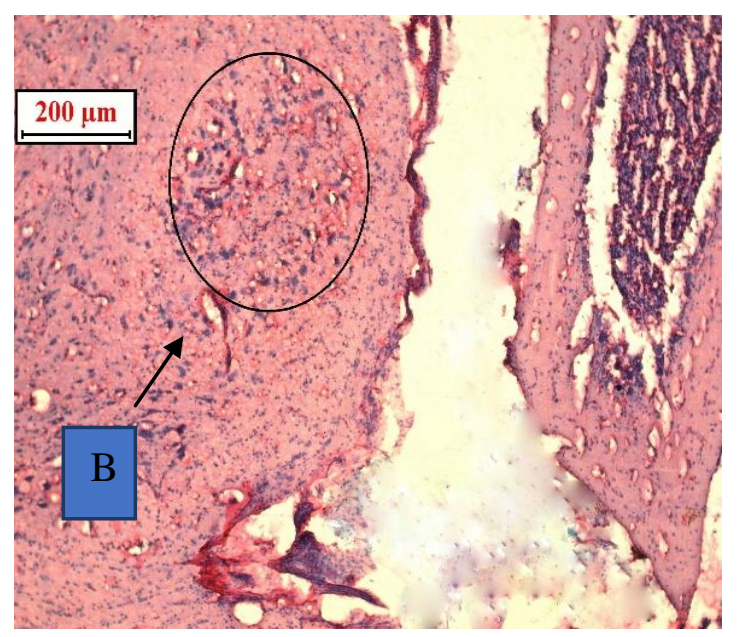

Figure.5 Photomicrograph showing LHN H\&E X 10. (B) LHN

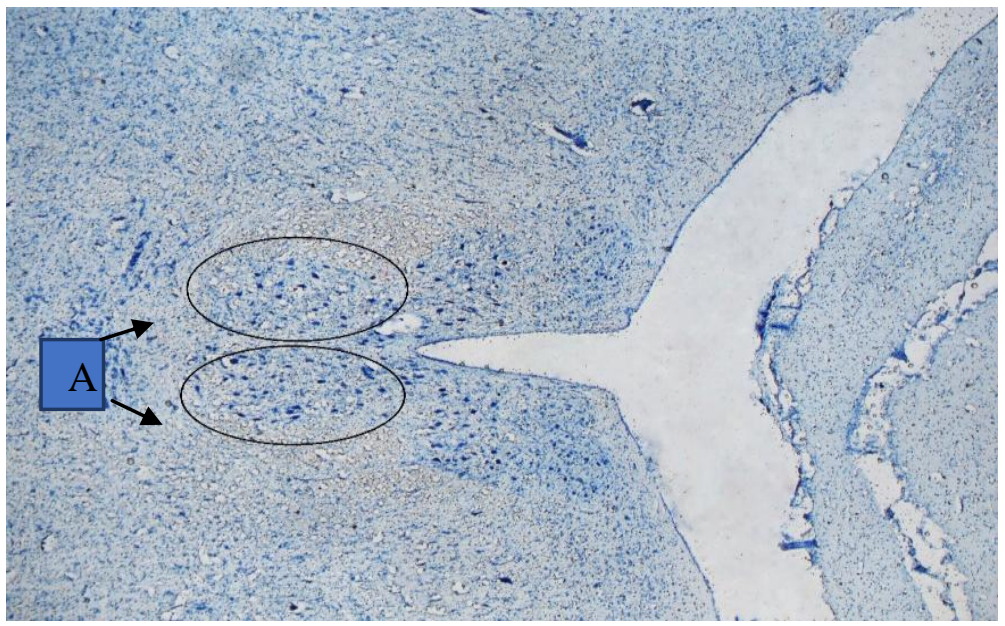

Figure.6 Photomicrograph showing PON in male chicken. Toluidine Blue X 4. (A) PON

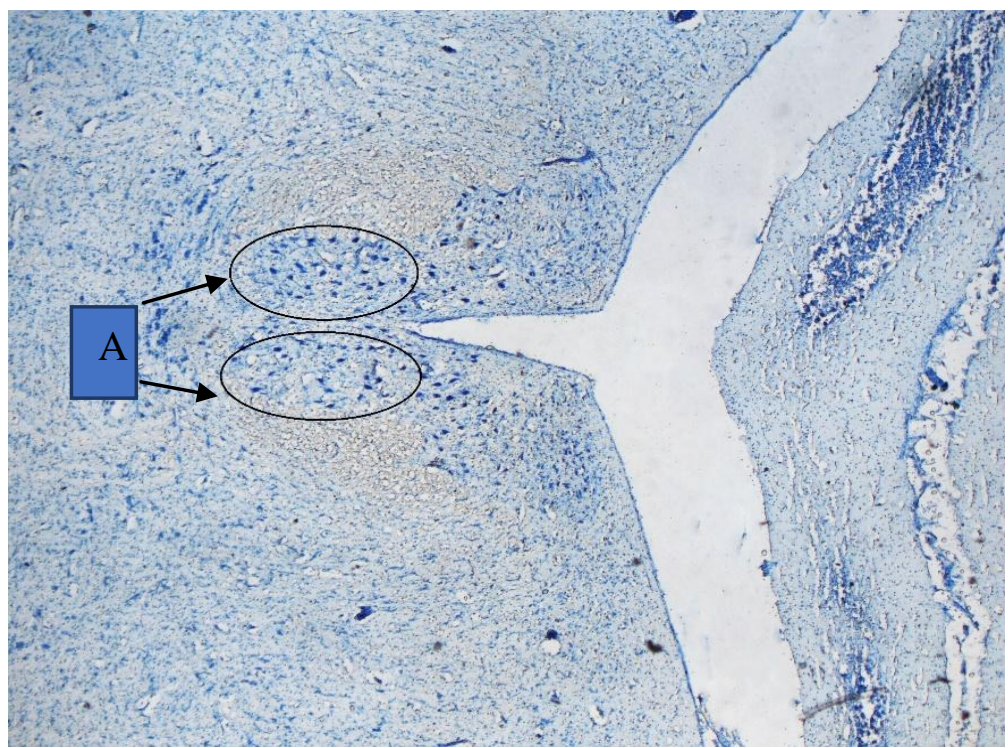

Figure.7 Photomicrograph showing PON in female Chicken, Toluidine Blue X 4. (A) PON 
In Nissl stained sections the PON appeared longer and more intensely stained in males than in females (Fig. 6 and 7). Similar observation was noted by Gorski et al., (1980) who stated that the volume of an intensely staining component of the preoptic area of the male rat was markedly larger than that of the female. The cell sizes and the size of the cell nuclei were also bigger in males. The average diameter of cell was 12.5 micron and 11.7 micron in male and female respectively.

In the present study nissle granules stained more intensely in male than female. Similar observation was cited by Uylings et al., (1984) who reported regarding the presence of (a) 5 times larger sexual dimorphic nucleus of the preoptic area (SDN-POA) in male rats as compared with female rats (b) Sex differences in dendritic size in the primate and rodent preoptic area.

Recently biochemical studies of the quail documented that sex differences in testosterone metabolism, aromatase activity and catecholamine content in pre-optic area (Schumacher and Balthazart, 1984; Ottinger et al, 1986). Due to these factors that Nissl substance stained more intensely in male than female probably indicates a higher intracellular metabolic activity.

Based on the anatomical findings presented here it can be concluded that there is a sex difference in the hypothalamic structure of chicken. It was observed that preoptic and medial suprachiasmatic nuclei were significantly larger in male than female. This study also indicated that Preoptic nucleus stained more intensely in males than in females, possibly differences in testosterone metabolism, aromatase activity and catecholamine content in pre-optic area which leads to a higher intracellular metabolic activity.

\section{Acknowledgements}

The authors are very much grateful to ViceChancellor, West Bengal University of Animal and Fishery Sciences; Dean, Faculty of Veterinary and Animal Sciences, WBUAFS and Head of the Department of Veterinary Anatomy and Histology, WBUAFS, Kolkata, India for providing necessary facilities.

\section{References}

Day, T. A., Willoughby, J. O., and Geffen, L. B. (1979). Thermoregulatory effects of preoptic area injections of noradrenaline in restrained and unrestrained rats. Brain research, 174(1), 175-179.

Gorski, R. A. (1968). The neural control of ovulation. In The Maternal Organism. pp. 1-66.

Gorski, R. A., Gordon, J. H., Shryne, J. E. and Southam, A. M. (1978). Evidence for a morphological sex difference within the medial preoptic area of the rat brain. Brain research, 148(2), 333346.

Gorski, R. A., Harlan, R. E., Jacobson, C. D., Shryne, J. E., and Southam, A. M. (1980). Evidence for the existence of a sexually dimorphic nucleus in the preoptic area of the rat. Journal of Comparative Neurology, 193(2), 529539.

Greenough, W. T., Carter, C. S., Steerman, C. and DeVoogd, T. J. (1977). Sex differences in dendritic patterns in hamster preoptic area. Brain Research, 126(1), 63-72.

Halasz, B. and Pupp, L. (1965). Hormone secretion of the anterior pituitary gland after physical interruption of all nervous pathways to the hypophysiotrophic area. Endocrinology, 77(3), 553-562. 
Ottinger, M. A., Schumacher, M., Clarke, R. N., Duchala, C. S., Turek, R. and Balthazart, J. (1986). Comparison of monoamine concentrations in the brains of adult male and female Japanese quail. Poultry science, 65(7), 1413-1420.

Panzica, G. C., Viglietti-Panzica, C. and Balthazart, J. (1996). The sexually dimorphic medial preoptic nucleus of quail: a key brain area mediating steroid action on male sexual behavior. Frontiers in neuroendocrinology, 17(1), 51-125.

Panzica, G., Viglietti- Panzica, C., Fiori, M. G., Calcagni, M., Anselmetti, G. and Balthazart, J. (1987). Cytoarchitectural analysis of the quail preoptic area. Evidence for a sex- related dimorphism in the medial preoptic nucleus. Italian Journal of Zoology, 54(1), 13-17.

Raisman, G. and Field, P. M. (1973). Sexual dimorphism in the neurophil of the preoptic area of the rat and its dependence on neonatal androgen. Brain research, 54, 1-29.

Robinson, S. M., Fox, T. O., Dikkes, P. and Pearlstein, R. A. (1986). Sex differences in the shape of the sexually dimorphic nucleus of the preoptic area and suprachiasmatic nucleus of the rat: 3-D computer reconstructions and morphometrics. Brain research, 371(2), 380-384.

Schumacher, M. and Balthazart, J. (1984). Sexual dimorphism in the hypothalamic metabolism of testosterone in the Japanese quail (Coturnixcoturnix japonica). In
Progress in brain research, 61, 53-61.

Squires, R.D. and Jacobson, F.H. (1968).

Chronic deficits of temperature regulation produced in cats by preoptic lesions. American Journal of Physiology-Legacy Content, 214(3), 549-560.

Suvarna, K. S., Layton, C. and Bancroft, J. D. (2012). Bancroft's Theory and Practice of Histological Techniques EBook. Elsevier Health Sciences.

Swanson, L. W., Kucharczyk, J. and Mogenson, G. J. (1978). Autoradiographic evidence for pathways from the medial preoptic area to the midbrain involved in the drinking response to angiotensin II. Journal of Comparative Neurology, 178(4), 645-659.

Uylings, H. B. M., Van Eden, C. G. and Verwer, R. W. H. (1984). Morphometric methods in sexual dimorphism research on the central nervous system. Progress in brain research, 61, 215-222.

Watson, J. T. and Adkins-Regan, E. (1989). Neuroanatomical Localization of Sex Steroid-Concentrating Cells in the Japanese Quail (Coturnix japonica); Autoradiography with $[3 \mathrm{H}]-$ Testosterone, $[3 \mathrm{H}]$-Estradiol, and $[3 \mathrm{H}]-$ Dihydrotestosterone. Neuroendocrinology, 49(1), 51-64.

Wiegand, S. J. and Terasawa, E. I. (1982). Discrete lesions reveal functional heterogeneity of suprachiasmatic structures in regulation of gonadotropin secretion in the female rat. Neuroendocrinology, 34(6), 395404.

\section{How to cite this article:}

Arkaprabha Shee, Partha Das, Sagun Saren, Palash Biswas, Aniruddha Banerjee and Md. Mofijul Islam. 2018. Evaluation of Sexual Dimorphism in Hypothalamus of Chicken (Gallus domesticus). Int.J.Curr.Microbiol.App.Sci. 7(07): 225-231.

doi: https://doi.org/10.20546/ijcmas.2018.707.027 